\title{
Sex-specific differences in white matter microvascular integrity after ischaemic stroke
}

\author{
Mark R Etherton (D) , ${ }^{1}$ Ona Wu, ${ }^{1,2,3}$ Pedro Cougo, ${ }^{1,4}$ Svetlana Lorenzano, ${ }^{1,5}$ Hua Li, ${ }^{3}$ \\ Lisa Cloonan, ${ }^{1}$ Mark J R J Bouts, ${ }^{1,2,6}$ Arne Lauer, ${ }^{1,7}$ Ken Arai, ${ }^{3}$ Eng H Lo, ${ }^{1,3}$ \\ Steve K Feske, ${ }^{8}$ Karen L Furie, ${ }^{9}$ Natalia S Rost ${ }^{1}$
}

To cite: Etherton MR, Wu 0, Cougo P, et al. Sex-specific differences in white matter microvascular integrity after ischaemic stroke. Stroke \& Vascular Neurology 2019;4: e000268. doi:10.1136/svn2019-000268

Received 22 July 2019 Revised 7 August 2019 Accepted 12 August 2019 Published Online First 3 September 2019

\section{Check for updates}

C Author(s) (or their employer(s)) 2019. Re-use permitted under CC BY-NC. No commercial re-use. See rights and permissions. Published by BMJ.

For numbered affiliations see end of article.

Correspondence to Dr Mark R Etherton; metherton@partners.org

\section{ABSTRACT}

Background and purpose Functional outcomes after ischaemic stroke are worse in women, despite adjusting for differences in comorbidities and treatment approaches. White matter microvascular integrity represents one risk factor for poor long-term functional outcomes after ischaemic stroke. The aim of the study is to characterise sex-specific differences in microvascular integrity in individuals with acute ischaemic stroke.

Methods A retrospective analysis of subjects with acute ischaemic stroke and brain MRI with diffusion-weighted (DWI) and dynamic-susceptibility contrast-enhanced (DSC) perfusion-weighted imaging obtained within 9 hours of last known well was performed. In the hemisphere contralateral to the acute infarct, normal-appearing white matter (NAWM) microvascular integrity was measured using the $K_{2}$ coefficient and apparent diffusion coefficient (ADC) values. Regression analyses for predictors of $K_{2}$ coefficient, DWI volume and good outcome (90-day modified Rankin scale (mRS) score $<2$ ) were performed.

Results 105 men and 79 women met inclusion criteria for analysis. Despite no difference in age, women had increased NAWM $K_{2}$ coefficient (1027.4 vs $692.7 \times 10^{-6} / \mathrm{s} ; p=0.006$ ). In women, atrial fibrillation $(\beta=583.6 ; p=0.04)$ and increasing NAWM ADC ( $\beta=4.4 ; p=0.02)$ were associated with increased NAWM $K_{2}$ coefficient. In multivariable regression analysis, the $K_{2}$ coefficient was an independent predictor of DWI volume in women ( $\beta=0.007 ; p=0.01$ ) but not men.

Conclusions In women with acute ischaemic stroke, increased NAWM $K_{2}$ coefficient is associated with increased infarct volume and chronic white matter structural integrity. Prospective studies investigating sex-specific differences in white matter microvascular integrity are needed.

\section{INTRODUCTION}

Sex disparities in acute ischaemic stroke (AIS) outcomes represent an important clinical issue. Compared with men, women have worse functional outcomes. ${ }^{12}$ Women are known to be older at the time of stroke, ${ }^{3}$ have higher rates of antecedent disability and atrial fibrillation, ${ }^{2}$ and lower rates of treatment with thrombolytic therapy. ${ }^{4}$ Even after adjusting for these differences, however, functional outcomes are worse in women. ${ }^{5}$ Understanding the factors that contribute to this sex disparity in stroke outcomes represents an opportunity to improve patient care and stroke outcomes.

The decreased structural integrity of cerebral white matter and increased blood-brain barrier (BBB) permeability are factors that negatively affect poststroke outcomes. ${ }^{6-9}$ Evidence of cerebral microvascular dysfunction can be detected on T2-weighted MRI as white matter hyperintensities (WMH), enhanced BBB permeability on perfusion-weighted imaging (PWI), or measured in normal-appearing white matter (NAWM) as loss of microstructural integrity on diffusion-tensor imaging (DTI), and reflected as an increase in the apparent diffusion coefficient (ADC) values. ${ }^{710}$ Both increased diffusivity and BBB permeability have been linked to poor outcomes after AIS, ${ }^{6-8}$ with a potential sexspecific contribution. ${ }^{11}$ We have also reported a novel association between increasing NAWM $K_{2}$ coefficient, as a potential surrogate of BBB permeability, and worse functional outcomes after AIS. ${ }^{7}$ Two important findings were observed in that analysis: (1) increased NAWM $K_{9}$ coefficient in women compared with men and (2) increased matrix metalloproteinase-2 (MMP-2) levels, as a measure of acute BBB disruption, ${ }^{12}$ were associated with worse longterm outcomes. The underlying aetiology of these sex-specific differences in BBB permeability and influences on ischaemic tissue and functional outcomes, however, was unclear.

In this study, we evaluated sex-specific clinical and radiographic variables associated with NAWM $K_{2}$ coefficient and their impact on infarct volume and 90-day functional outcomes after AIS.

\section{METHODS}

Standard protocol approvals, registrations and patient consents

This study and the use of human participants was approved by the local Institutional Review Board. Written informed consent was obtained from all participating subjects or their surrogates. 


\section{Subjects and inclusion/exclusion criteria}

We performed a retrospective analysis of a prospective biomarker cohort study of consecutive adult AIS subjects admitted between February 2007 and 2011 (National Institutes of Health Specialized Program of Translational Research in Acute Stroke). ${ }^{13} 14$

All subjects presenting to two academic medical centres within 9 hours of the last known well (LKW) and with signs and symptoms of AIS were screened for eligibility. To be eligible for inclusion in this study, subjects were required to undergo brain MRI within 9 hours from LKW containing diffusion-weighted imaging (DWI), dynamicsusceptibility contrast (DSC) perfusion-weighted MRI, and fluid-attenuated inversion recovery (FLAIR) MRI sequences; have a confirmed acute infarct on DWI; and have plasma MMP-2 levels collected at the time of study enrollment. We excluded patients with artefactual MMP-2 recordings and those with acute infarcts in both hemispheres.

\section{Clinical assessments}

Age, medical history and antecedent medications were obtained from the subject or their surrogates or otherwise from the review of the medical record at the time of admission. Admission systolic and diastolic blood pressures were acquired from the first documented vital signs. Treatment with intravenous alteplase was recorded. Tobacco use was dichotomised as current or past use versus never. Alcohol use was defined and dichotomised as current versus none. Anticoagulant use was defined as prehospital use of lovenox or coumadin.

\section{Imaging analysis}

MRI protocol

The majority of MRIs were performed on a $1.5 \mathrm{~T}$ General Electric Signa HDx scanner with the following acquisition parameters. DTI: repetition time (TR)/echo time $(\mathrm{TE})=5000 / 85.3 \mathrm{~ms}, 220 \mathrm{~mm}$ field-of-view, $5 \mathrm{~mm}$ slice thickness with $1 \mathrm{~mm}$ gap, in-plane resolution of $0.86 \times 0.86 \mathrm{~mm}^{2}(128 \times 128$ acquisition matrix up-sampled to $256 \times 256$ ), three $0 \mathrm{~s} / \mathrm{mm}^{2}$ (b-zero) and $1000 \mathrm{~s} / \mathrm{mm}^{2}$ in 25 directions. FLAIR: TR/TE/TI=10 002/145/2200 ms, $220 \mathrm{~mm}$ field-of-view, $5 \mathrm{~mm}$ slice thickness with $1 \mathrm{~mm}$ gap, and in-plane resolution of $0.86 \times 0.86 \mathrm{~mm}^{2}$. For 10 subjects, FLAIR scans were acquired at a separate time from the original DWI and DSC sequences due to an unavailable or unusable FLAIR scan on the original MRI. DSC: 80 acquisitions, TR/TE $=1500 / 40 \mathrm{~ms}, 220 \mathrm{~mm}$ field-of-view, $5 \mathrm{~mm}$ slice thickness with $1 \mathrm{~mm}$ gap, $1.72 \times 1.72 \mathrm{~mm}^{2}$ in-plane resolution. DSC sequences were acquired using bolus tracking of a single dose of gadolinium-based contrast agent $(0.1 \mathrm{mmol} / \mathrm{kg})$ administered with a power injector. Motion and eddy current distortions were corrected in all DTI datasets and the ADC maps were then derived from the high-b-value and low-b-value DTI sequences. ${ }^{15}$

To estimate BBB permeability, the $K_{2}$ coefficient $(/ \mathrm{s})$ was mathematically derived from the concentration-time curves using the Boxerman-Weisskoff model. ${ }^{16}$ In brief, the effective transverse relaxation rate due to the contrast agent, a parameter representing the per voxel concentration of contrast agent, is represented as:

$$
\Delta \bar{R}_{2}^{*}(t) \approx K_{1} \cdot \Delta \bar{R}_{2}^{*}(t)-K_{2} \int_{0}^{1} \Delta \bar{R}_{2}^{*}\left(t^{\prime}\right) d t^{\prime}
$$

A least-squares fit to this equation can subsequently be used to solve for the $K_{2}$ coefficient on a voxelwise basis.

Mean transit time (MTT) maps were calculated from deconvolved DSC data converted to concentration changes over time using a tracer arrival timing-insensitive technique. ${ }^{1718}$

\section{Volumetric lesion mapping}

The DWI and MTT lesions were outlined using an intensity threshold-based method. All DWI lesions were crossreferenced to ADC maps to exclude subacute and chronic infarcts as well as infarcts involving the bilateral cerebral hemispheres. The acute infarct volume and ipsilateral perfusion deficit, assessed on DWI sequences (DWIv) and MTT maps (MTTv), respectively, were calculated semiautomatically using MRIcro software (University of Nottingham School of Psychology, Nottingham, UK; www. mricro.com).

WMH lesions in the hemisphere contralateral to the acute infarct were outlined in a semi-automated manner using MRIcro software as described previously. ${ }^{11} 19$ All images were analysed by trained research staff that were blinded to all outcome data.

\section{NAWM segmentation and analysis}

NAWM segmentation in the contralateral hemisphere was performed as described previously (figure 1$).{ }^{7}$ The mean ADC and $K_{2}$ coefficients were measured in contralateral NAWM as defined by the probabilistic mask.

Stroke and outcomes assessment

All subjects were evaluated at the time of admission by a trained neurologist and stroke severity was determined using the National Institutes of Health Stroke Scale (NIHSS). Stroke subtype was categorised according to the Causative Classification of Stroke System (CCS) score. ${ }^{20}$ Haemorrhagic transformation was defined as the presence of any petechial or parenchymal haematoma on follow-up CT or MRI (obtained within 48 hours of LKW). Functional outcome at 90 days was measured using the mRS score, which was obtained either through an in-person or validated telephone-based interview. ${ }^{21}$ Good functional outcome was defined as a 90-day mRS score of $<2$.

\section{Statistical analysis}

WMH volume (WMHv) and DWI volume (DWIv) were normalised to the intracranial area. ${ }^{22}$ Univariable analysis was performed using linear regression to determine factors associated with $K_{2}$ coefficient and DWIv and logistic regression to evaluate factors associated with good functional outcome. Variables that displayed an association, a priori set at $\mathrm{p}<0.05$, in univariable analysis were inserted into a backward stepwise elimination 


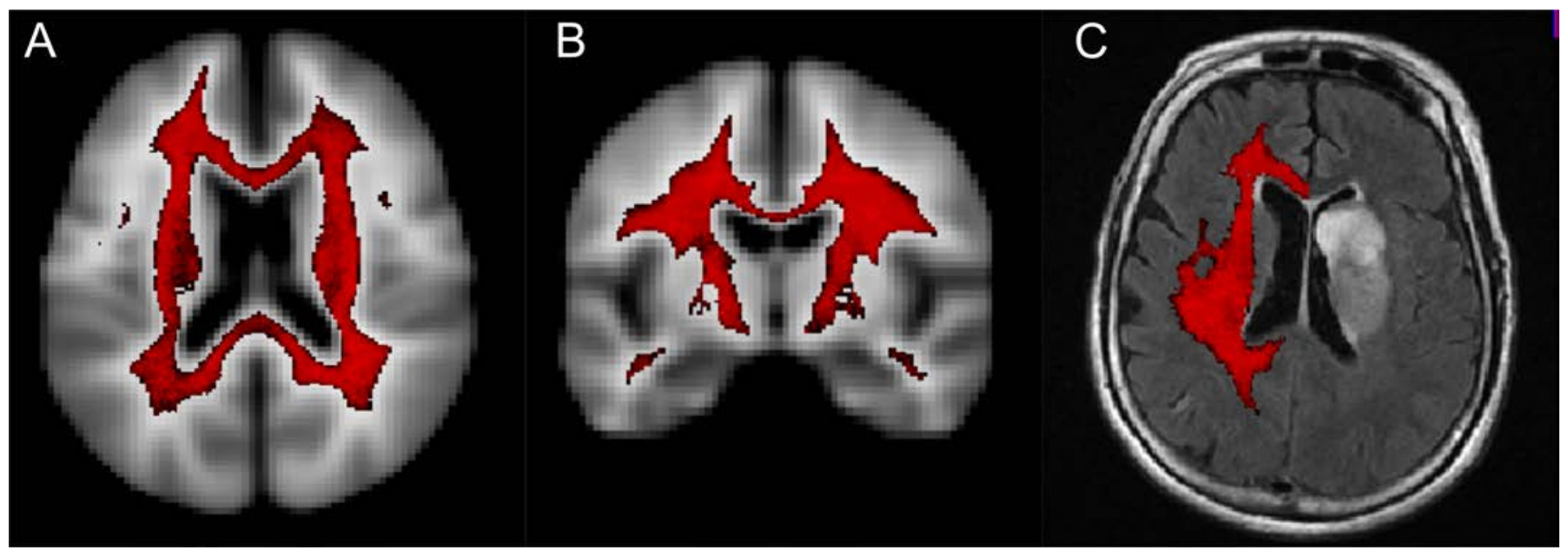

Figure 1 NAWM segmentation. (A) Axial and (B) coronal views of probabilistic white matter mask with threshold of $>95 \%$ probability white matter (red) superimposed on the International Consortium for Brain Mapping 425 atlas. (C) Representative image of final contralesional normal-appearing white matter mask (red) overlaid on FLAIR image.

multivariable regression model. To minimise influences of collinearity, WMHv and NAWM ADC, as markers of global structural integrity, were input into separate multivariable regression models when significant in univariable analysis. Admission NIHSS was excluded from multivariable regression models for DWIv and good outcomes due to the known strong association between NIHSS and DWIv. ${ }^{23}$ Statistical significance was set at a $p$ value of $<0.05$.

\section{RESULTS}

A total of 105 men and 79 women met inclusion criteria for analysis in this study. The baseline demographics are shown in table 1 . Women were more likely to have atrial fibrillation and cardioembolic strokes. Men had a greater incidence of hyperlipidaemia and strokes attributed to large-artery atheroembolism. Treatment rates with intravenous alteplase were similar between men and women and only one patient in the cohort underwent intraarterial therapy. There was no difference in WMHv, DWIv, MTT volume or NAWM ADC between men and women. Women, however, had significantly greater NAWM $K_{2}$ coefficient (table 1).

In women, WMHv, NAWM ADC and atrial fibrillation were associated with greater NAWM $K_{2}$ coefficient in univariable linear regression analysis (table 2). In multivariable analyses, increasing NAWM ADC was associated with increased NAWM $K_{2}$ coefficient in women, whereas $\mathrm{WMHv}$ was not (table 3). In contrast, in men, WMHv and age were associated with NAWM $K_{2}$ coefficient in univariable linear regression but only WMHv remained an independent predictor in the multivariable analysis (table 3). In the entire cohort, we observed no association between strokes attributed to large-artery atheroembolism ( $\beta=75.0 ; p=0.84)$ or cardioembolism $(\beta=163.1$; $\mathrm{p}=0.58)$ and the NAWM $K_{2}$ coefficient.

In both men and women, the admission NIHSS was a significant predictor of DWIv in univariable analysis (table 4). In women, administration of intravenous alteplase, haemorrhagic transformation and increasing NAWM $K_{2}$ coefficient were independently associated with DWIv. In comparison, in men, only atrial fibrillation was associated with increased DWIv. Subsequent linear regression analysis including sex, NAWM $K_{2}$ coefficient and an interaction term (male sex $\bullet$ NAWM $K_{2}$ coefficient) showed that NAWM $K_{2}$ coefficient $(\beta=0.006$; $\mathrm{p}=0.037)$ and the interaction term $(\beta=-0.007 ; \mathrm{p}=0.027)$ were independent predictors of DWIv for the entire cohort.

In multivariable logistic regression analysis, increasing NAWM ADC was an independent predictor of the decreased likelihood of good outcome for both sexes (table 5).

\section{DISCUSSION}

We report that the NAWM $K_{2}$ coefficient, as a measure of microvascular integrity, is significantly increased in women compared with men with AIS. In addition, in women we observe sex-specific relationships: NAWM $K_{2}$ coefficient is independently associated with NAWM ADC values and a predictor of infarct volume. These findings suggest that increased NAWM $K_{2}$ coefficient in women, as a surrogate of BBB dysfunction, is associated with increased infarct size, which predisposes to worse longterm outcomes after stroke.

Our finding of sex-specific differences in NAWM $K_{2}$ coefficient, as a putative measure of BBB dysfunction, is of clinical interest. We have previously reported an association between NAWM structural integrity (fractional anisotropy) and sex-specific functional outcomes. ${ }^{11}$ Together, these observations offer some insight into the potential factors contributing to the disparity in stroke outcomes between sexes as they suggest that sex-specific differences in NAWM microvascular integrity predispose to worse outcomes in women. There are several potential explanations for the observed sex-specific differences in 
Table 1 Comparison of admission and clinical variables in men and women with AIS

\begin{tabular}{|c|c|c|c|}
\hline Variable & $\begin{array}{l}\text { Women } \\
(\mathrm{N}=79)\end{array}$ & $\begin{array}{l}\text { Men } \\
(\mathrm{N}=105)\end{array}$ & $P$ values \\
\hline Age, years, mean (SD) & $68.5(16.3)$ & $68.1(14.0)$ & 0.55 \\
\hline \multicolumn{4}{|l|}{ Medical history, n (\%) } \\
\hline Anticoagulant use & $10(12.7)$ & $19(18.1)$ & 0.43 \\
\hline Atrial fibrillation & $29(36.7)$ & $21(20.0)$ & 0.02 \\
\hline Coronary artery disease & $15(19.0)$ & $29(27.6)$ & 0.24 \\
\hline Diabetes & $13(16.5)$ & $25(23.8)$ & 0.30 \\
\hline Hyperlipidaemia & 27 (34.2) & $55(52.4)$ & 0.02 \\
\hline Hypertension & $52(65.8)$ & 77 (73.3) & 0.35 \\
\hline Prior stroke/TIA & $18(22.8)$ & $26(24.8)$ & 0.89 \\
\hline \multicolumn{4}{|l|}{ CCS subtype classification, n (\%) } \\
\hline Cardioembolic & $48(60.8)$ & $46(43.8)$ & 0.03 \\
\hline Large artery & $6(7.6)$ & $33(31.4)$ & $<0.001$ \\
\hline Small vessel & $4(5.1)$ & $5(4.8)$ & 1 \\
\hline Other/Undetermined & $21(26.6)$ & $20(19.0)$ & 0.30 \\
\hline \multicolumn{4}{|l|}{ Clinical variables } \\
\hline Admission NIHSS, median (IQR) & $6(3,12)$ & $5(2,11)$ & 0.05 \\
\hline Body mass index, $\mathrm{kg} / \mathrm{m}^{2}$, mean (SD) & $27.8(6.5)$ & $28.3(5.5)$ & 0.52 \\
\hline Systolic blood pressure, mm Hg, median (IQR) & $148(136-173)$ & $158(140-174)$ & 0.14 \\
\hline Diastolic blood pressure, mm Hg, median (IQR) & $84(72-92)$ & $84(76-89)$ & 0.83 \\
\hline MMP-2 level, ng/mL, median (IQR) & $304.6(242.7-372.1)$ & $301.7(237.8-369.7)$ & 0.80 \\
\hline IV alteplase treatment, $\mathrm{n}(\%)$ & $30(38.0)$ & 35 (33.3) & 0.62 \\
\hline \multicolumn{4}{|l|}{ Radiographic variables } \\
\hline Haemorrhagic transformation, $\mathrm{n}(\%)$ & $16(27.1)$ & $24(22.9)$ & 0.79 \\
\hline Parenchymal haematoma, n (\%) & $5(5.9)$ & $11(11.1)$ & 0.46 \\
\hline WMHv, $\mathrm{cm}^{3}$, median (IQR) & $2.0(0.8-5.5)$ & $1.7(0.9-3.8)$ & 0.55 \\
\hline DWIv, $\mathrm{cm}^{3}$, median (IQR) & $6.9(1.2-30.2)$ & $7.5(1.7-21.9)$ & 0.76 \\
\hline MTTv, $\mathrm{cm}^{3}$, median (IQR) & $32.1(5.3-81.0)$ & $23.5(5.8-78.2)$ & 0.92 \\
\hline ADC $\left(\times 10^{-6}\right)$ NAWM, $\mathrm{mm}^{2} / \mathrm{s}$, mean (SD) & $804.0(69.2)$ & $791.9(48.7)$ & 0.19 \\
\hline$K_{2}$ coefficient $\left(\times 10^{-6}\right)$ NAWM, $/ \mathrm{s}$, mean (SD) & $1027.4(1192.9)$ & $692.7(2460.9)$ & 0.006 \\
\hline NAWM mask volume, $\mathrm{cm}^{3}$, median (IQR) & $44.0(35.4-51.5)$ & $53.4(43.5-63.1)$ & $<0.001$ \\
\hline Time from last seen well to MRI, min, median (IQR) & $346(256-438)$ & $345.5(250.8-437.8)$ & 0.90 \\
\hline \multicolumn{4}{|l|}{ Functional outcomes } \\
\hline 90-day good outcome (mRS <2), n (\%) & $29(46.8)$ & $55(58.5)$ & 0.20 \\
\hline
\end{tabular}

Bold values indicate $P$ value $<0.05$.

ADC, apparent diffusion coefficient; AIS, acute ischaemic stroke; CCS, causative classification system; DWIv, diffusion-weighted imaging volume; IQR, interquartile range; IV, intravenous; MMP, matrix metalloproteinaise; MTTv, mean transit time volume; NAWM, normal-appearing white matter; NIHSS, National Institutes of Health Stroke Scale; TIA, transient ischemic attack; WMHv, white matter hyperintensity volume.

NAWM $K_{\mathrm{q}}$ coefficient. First, we observed no substantial difference in age, hypertension, diabetes, anticoagulant use, intravenous alteplase treatment, rate of haemorrhagic transformation, WMHv or stroke severity in women. A relationship between NAWM BBB permeability and white matter structural integrity has been reported in individuals with cerebral small-vessel disease. ${ }^{25-27}$ In our acute cohort, however, we observed no difference in strokes attributed to small-vessel occlusive disease between sexes.
We also observed no difference in baseline MMP-2 levels between sexes, a molecule thought to play a role in BBB remodelling. ${ }^{7}$ Another study showed that diastolic blood pressure and blood glucose were independently associated with BBB permeability abnormalities, ${ }^{28}$ however, we observed no differences in admission blood pressure or rates of hypertension between sexes. Another hypothesis is the potential hormonal dysregulation of the BBB in women. Different murine models of oestrogen depletion 
Table 2 Univariable analysis of sex-specific associations with NAWM $K_{2}$ coefficient

\begin{tabular}{|c|c|c|c|c|}
\hline \multirow[b]{2}{*}{ Variable } & \multicolumn{2}{|l|}{ Women } & \multicolumn{2}{|l|}{ Men } \\
\hline & Estimate & $P$ values & Estimate & $P$ values \\
\hline Age & 11.3 & 0.17 & 36.0 & 0.04 \\
\hline BMI & -41.8 & 0.06 & -64.0 & 0.20 \\
\hline Anticoagulant use & 523.8 & 0.20 & 2.9 & 0.99 \\
\hline Atrial fibrillation & 583.6 & 0.04 & -935.3 & 0.12 \\
\hline Diabetes & -118.6 & 0.75 & 603.2 & 0.29 \\
\hline Hyperlipidaemia & 82.6 & 0.77 & 613.2 & 0.20 \\
\hline Hypertension & -123.4 & 0.67 & -362.2 & 0.51 \\
\hline Prior stroke/TIA & 336.7 & 0.30 & 650.6 & 0.24 \\
\hline Cardioembolic stroke & 194.6 & 0.48 & 66.7 & 0.89 \\
\hline Admission NIHSS & 40.4 & 0.07 & 31.4 & 0.40 \\
\hline Systolic blood pressure & 0.9 & 0.86 & 8.0 & 0.36 \\
\hline MMP-2 level & 0.4 & 0.69 & 1.4 & 0.45 \\
\hline IV alteplase treatment & 195.5 & 0.48 & -732.6 & 0.15 \\
\hline HT & 413.4 & 0.17 & 357.1 & 0.52 \\
\hline WMHv & 31.8 & 0.04 & 131.1 & 0.005 \\
\hline NAWM ADC & 4.4 & 0.02 & 2.2 & 0.66 \\
\hline NAWM mask volume & -17.8 & 0.11 & -24.0 & 0.13 \\
\hline
\end{tabular}

Bold values indicate $P$ value $<0.05$

ADC, apparent diffusion coefficient; BMI, body mass index; HT, haemorrhagic transformation; IV, intravenous; MMP, matrix metalloproteinase; NAWM, normal-appearing white matter; NIHSS, National Institutes of Health Stroke Scale; TIA, transient ischemic attack; WMHv, white matter hyperintensity volume.

have shown an association with increased BBB permeability. ${ }^{29}{ }^{30}$ These observations could suggest that in our female patient population, which is likely predominantly postmenopausal based on age, relative oestrogen deficiency contributes to increased NAWM $K_{2}$ coefficient. Future studies investigating the association between menopausal status and BBB permeability could therefore be informative.

We also demonstrate an association between increasing white matter structural injury and alterations in NAWM BBB permeability. In both men and women, increased WMH burden is associated with NAWM $K_{2}$ coefficient.

Table 3 Multivariable backward stepwise elimination linear regression analysis for sex-specific predictors of NAWM $K_{2}$ coefficient

\begin{tabular}{|c|c|c|c|c|}
\hline & Model & Variables & Estimate & $\begin{array}{l}P \\
\text { values }\end{array}$ \\
\hline \multirow[t]{4}{*}{ Women } & 1 & Atrial fibrillation & - & - \\
\hline & & NAWM ADC & 4.4 & 0.02 \\
\hline & 2 & Atrial fibrillation & 468.5 & 0.10 \\
\hline & & WMHv & 25.1 & 0.11 \\
\hline \multirow[t]{2}{*}{ Men } & 1 & Age & 27.3 & 0.11 \\
\hline & & WMHv & 115.7 & 0.01 \\
\hline
\end{tabular}

Bold values indicate $P$ value $<0.05$.

ADC, apparent diffusion coefficient; NAWM, normal-appearing white matter; WMHv, white matter hyperintensity volume.
These findings support the hypothesis that early microvascular dysfunction in NAWM, causing BBB leakage, precedes white matter damage and WMH development. Corroborating this hypothesis, in patients with the smallvessel disease, increased BBB permeability is associated with WMH burden and hypertension. ${ }^{31}$ We go on to show in women that NAWM ADC, a marker of altered water mobility, is associated with increased BBB permeability. These findings suggest that there may be sex-specific differences in the early relationship between NAWM BBB permeability and abnormal water mobility or structural integrity, which represents a risk factor for incident stroke and poor outcomes. ${ }^{8} 2732$

In women, NAWM $K_{2}$ coefficient is associated with larger acute infarct volume. This occurred despite no difference between groups in age, intravenous alteplase administration, small-vessel occlusive stroke subtype, WMH burden and rates of haemorrhagic transformation. One interpretation of these results is that increased NAWM $K_{2}$ coefficient predisposes, or is a surrogate, for larger acute infarct volume. In support of this hypothesis, prior work in patients with AIS has shown that the $K_{2}$ coefficient of the DWI lesion is inversely associated with rates of reperfusion post alteplase,$^{33}$ and positively associated with the DWI volume and severity of intracranial haemorrhage postendovascular thrombectomy. ${ }^{34}$ Based on what has been shown previously on the relationship of increasing $\mathrm{BBB}$ permeability and poor functional 
Table 4 Sex-specific associations with acute infarct volume

\begin{tabular}{|c|c|c|c|c|c|c|}
\hline \multirow[b]{3}{*}{ Variable } & \multicolumn{4}{|l|}{ Women } & \multirow{2}{*}{\multicolumn{2}{|c|}{$\begin{array}{l}\text { Men } \\
\text { Univariable }\end{array}$}} \\
\hline & \multicolumn{2}{|c|}{ Univariable } & \multicolumn{2}{|c|}{ Multivariable } & & \\
\hline & Estimate & P values & Estimate & $P$ values & Estimate & $P$ values \\
\hline Age & -0.1 & 0.60 & & & 0.3 & 0.20 \\
\hline BMI & -0.55 & 0.25 & & & -0.84 & 0.21 \\
\hline Anticoagulant use & -4.5 & 0.61 & & & -6.5 & 0.45 \\
\hline Atrial fibrillation & -1.2 & 0.84 & & & 20.8 & 0.01 \\
\hline Diabetes & -11.8 & 0.14 & & & -3.6 & 0.64 \\
\hline Hyperlipidaemia & 0.6 & 0.92 & & & -6.4 & 0.34 \\
\hline Hypertension & -5.7 & 0.36 & & & 12.8 & 0.09 \\
\hline Prior stroke/TIA & 6.1 & 0.38 & & & 13.7 & 0.07 \\
\hline Cardioembolic stroke & 1.8 & 0.76 & & & -1.1 & 0.87 \\
\hline Admission NIHSS & 2.7 & $<0.001$ & & & 3.0 & $<0.001$ \\
\hline Systolic blood pressure & -0.1 & 0.48 & & & 0.02 & 0.85 \\
\hline IV alteplase treatment & 13.3 & 0.03 & 12.6 & 0.03 & 7.8 & 0.27 \\
\hline HT & 20.6 & 0.005 & 16.9 & 0.01 & 13.0 & 0.11 \\
\hline WMHV & 0.3 & 0.43 & & & 0.13 & 0.84 \\
\hline NAWM ADC & 0.02 & 0.66 & & & 0.08 & 0.24 \\
\hline NAWM $K_{2}$ & 0.006 & 0.01 & 0.007 & 0.01 & -0.001 & 0.50 \\
\hline NAWM mask volume & 0.15 & 0.54 & & & -0.1 & 0.78 \\
\hline
\end{tabular}

Multivariable Regression Equation.

Women : $D W I v=\beta_{0}+\beta_{1} \cdot(I V$ alteplase $)+\beta_{2} \cdot(H T)+\beta_{3} \cdot\left(N A W M K_{2}\right)$

Men: NA.

Bold values indicate $P$ value $<0.05$

ADC, apparent diffusion coefficient; BMI, body mass index; DWIv, diffusion-weighted imaging lesion volume; HT, haemorrhagic

transformation; IV, intravenous; NA, not applicable; NAWM, normal-appearing white matter; NIHSS, National Institutes of Health Stroke Scale;

$\mathrm{TIA}$, transient ischemic attack; WMHv, white matter hyperintensity volume.

outcomes after AIS, ${ }^{78} 35$ our findings are promising and hypothesis generating for a role of microvascular integrity in the sex-disparity of stroke outcomes.

There are several limitations of this study that warrant consideration. First, this was a retrospective analysis of a hospital-based biomarker study of AIS subjects who underwent a clinical MRI with PWI within 9 hours from LKW. Non-isotropic voxels were used for analysis of diffusivity anisotropy, which could raise the possibility of partial volume effects as confounders of the MRI measurements. ${ }^{36}$ This was also an analysis of clinical MRIs performed on patients with acute stroke where differences in patient head orientation, for example, could introduce registration errors of the automated NAWM mask. By automatically applying the same probabilistic white matter mask to each subject, however, this approach reduces human bias from manually placed region-of-interests. An automated approach for selecting NAWM regions-of-interest is therefore a clinically feasible method for measuring global NAWM microstructural and microvascular integrity in patients with AIS.

The interpretation of changes in the $K_{2}$ coefficient also warrants discussion as this approach is relatively new for measuring BBB permeability in AIS subjects. At the individual voxel level the $K_{0}$ coefficient is a representation of the contribution of the T1 leakage effects due to contrast agent kinetics and the T2* leakage effects due to the tissue microstructure. ${ }^{3738}$ For these reasons, the $K_{9}$ coefficient may reflect differences in other microvascular and tissue microstructural properties. The measurement of $K_{\mathrm{q}}$ in the acute setting is also likely a dynamic process with possible global influence from the acute ischaemic event. $^{39}$ As compared with dynamic contrast-enhanced (DCE) MRI and $K_{\text {trans }}$, the measurement of NAWM $K_{2}$ coefficient might have reduced sensitivity in the setting of delayed bolus arrival. However, by restricting our analyses to the hemisphere contralateral to the acute infarct we attempted to account for this limitation. We also cannot exclude the possibility that AIS can influence blood regulation and BBB permeability on remote sites or that differences in contralateral NAWM $K_{2}$ reflect differences in reactivity in the acute setting. A DSC-based approach has, however, previously been shown to be feasible for characterising BBB disruption in AIS $^{33} 34$ and there are distinct advantages of the DSC technique for this population. DCE-MRI has a long acquisition time, which limits its feasibility for studying AIS subjects. In contrast, DSC image acquisition takes 1-2 min. Several prior reports 
Table 5 Sex-specific associations with good outcomes

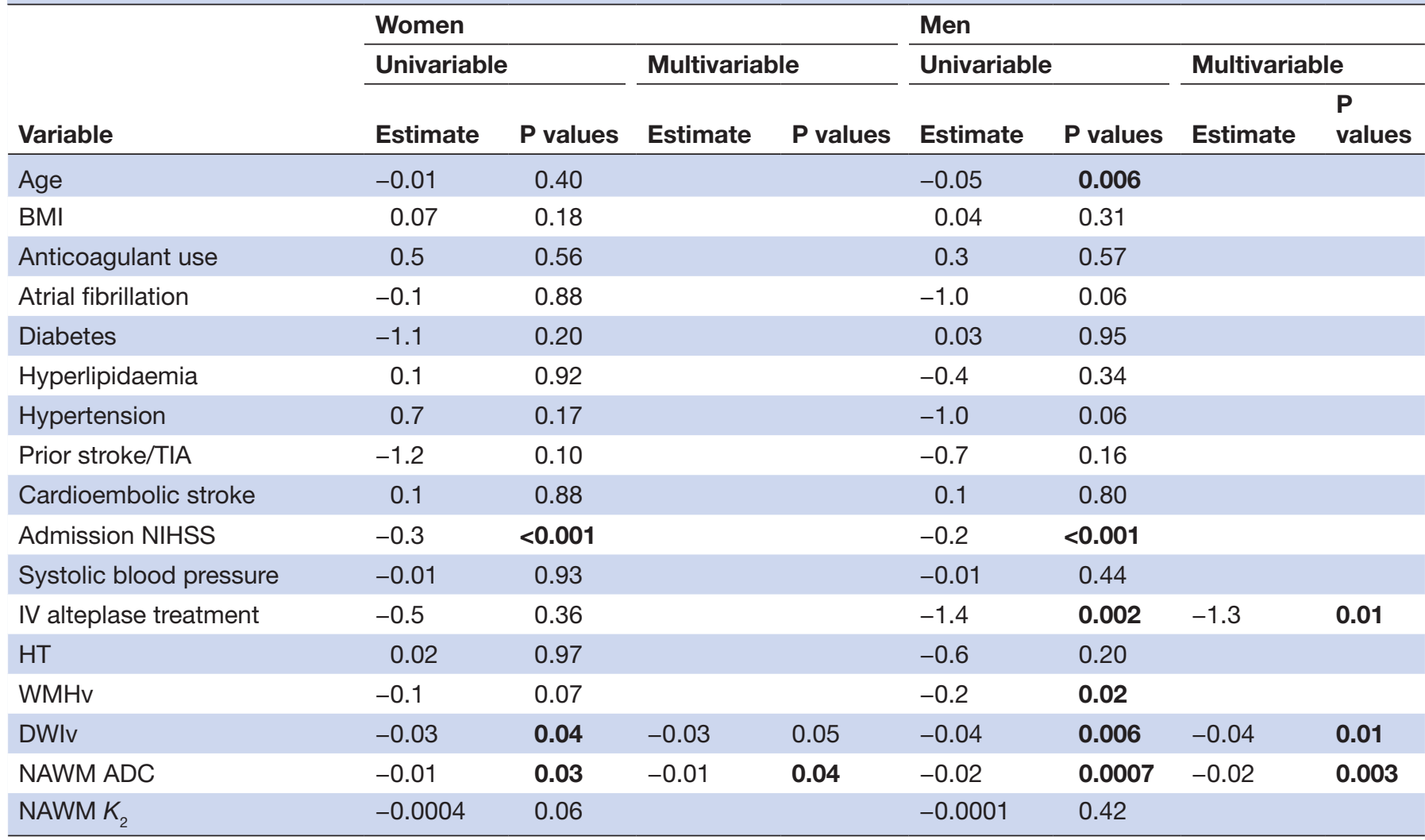

Multivariable Regression Equation.

Women : Good outcome $=\beta_{0}+\beta_{1} \cdot$ DWIV $+\beta_{2} \cdot($ NAW ADC $)$

Men : Good outcome $=\beta_{0}+\beta_{1} \cdot D W I V+\beta_{2} \cdot($ NAW ADC $)+\beta_{3} \cdot($ IV alteplase $)+\beta_{4} \cdot($ Age $)$

Bold values indicate $P$ value $<0.05$.

ADC, apparent diffusion coefficient; BMI, body mass index; DWlv, diffusion-weighted imaging lesion volume; HT, haemorrhagic

transformation; IV, intravenous; NAWM, normal-appearing white matter; TIA, transient ischemic attack; WMHv, white matter hyperintensity volume.

have also demonstrated a strong correlation between $\mathrm{K}_{2}$ and $\mathrm{K}_{\text {trans }}{ }^{38}{ }^{40}$ Lastly, although we show sex-specific differences in NAWM $K_{2}$ coefficient and the relationship with infarct volume, the aetiology of this observation is less clear. Future stroke studies applying DSC-MRI to characterise sex-specific differences in BBB permeability, stratified for comorbidities, are therefore necessary.

\section{CONCLUSIONS}

Despite no differences in age and stroke severity, the NAWM $K 2$ coefficient is increased in women with AIS and associated with infarct volume. These observations suggest that sex-specific differences in microvascular integrity may contribute to the sex disparity in poststroke outcomes.

\section{Author affiliations}

${ }^{1}$ Department of Neurology, Massachusetts General Hospital and Harvard Medical School, Boston, Massachusetts, USA

${ }^{2}$ Martinos Center for Biomedical Imaging, Massachusetts General Hospital,

Charlestown, MA, United States

${ }^{3}$ Department of Radiology, Massachusetts General Hospital and Harvard Medical

School, Boston, MA, United States

${ }^{4}$ Medical School of Ribeirao Preto, University of Sao Paulo, Sao Paulo, Brazil
${ }^{5}$ Department of Human Neurosciences, Sapienza University of Rome, Rome, Italy ${ }^{6}$ Institute of Psychology, Leiden University, Leiden, Netherlands

${ }^{7}$ Department of Neuroradiology, Goethe-Universitat Frankfurt am Main, Frankfurt, Germany

${ }^{8}$ Department of Neurology, Brigham and Women's Hospital and Harvard Medical School, Boston, MA, United States

${ }^{9}$ Department of Neurology, Warren Alpert Medical School of Brown University, Providence, Rhode Island, USA

Contributors MRE: designed and conceptualised study; analysed the data; data acquisition; drafted the manuscript for intellectual content. OW: designed and conceptualised study; analysed the data; data acquisition; revised the manuscript for intellectual content. PC, SL, HL, LC, MJRJB, AL, KA and EHL: data acquisition; revised manuscript for intellectual content. SKF and KLF: designed and conceptualised study; revised manuscript for intellectual content. NSR: designed and conceptualised study; analysed data; revised manuscript for intellectual content.

Funding This work was supported by the National Institutes of Health NINDS Specialized Program of Transitional Research in Acute Stroke grant P50-NS051343; R01NS082285; R01NS086905; R01NS059775. Mark Etherton is supported in part by AHA - 17CPOST33680102. Ona Wu is supported in part by NIH-NINDS P50NS051343, R01NS059775, R01NS063925, R01NS082285 and R01NS086905. Pedro Cougo, Svetlana Lorenzano, Hua Li, Steven Feske, and Karen Furie were supported by NIH 5P50NS051343. Natalia S. Rost is supported in part by NIHNINDS R01NS082285 and R01NS086905.

Competing interests None declared.

Patient consent for publication Not required. 
Provenance and peer review Not commissioned; externally peer reviewed. Data availability statement Data are available upon reasonable request.

Open access This is an open access article distributed in accordance with the Creative Commons Attribution Non Commercial (CC BY-NC 4.0) license, which permits others to distribute, remix, adapt, build upon this work non-commercially, and license their derivative works on different terms, provided the original work is properly cited, appropriate credit is given, any changes made indicated, and the use is non-commercial. See: http://creativecommons.org/licenses/by-nc/4.0/.

ORCID iD

Mark R Etherton http://orcid.org/0000-0002-4739-2449

\section{REFERENCES}

1. Appelros P, Stegmayr B, Terent A. Sex differences in stroke epidemiology: a systematic review. Stroke 2009;40:1082-90.

2. Phan HT, Blizzard CL, Reeves MJ, et al. Sex differences in longterm mortality after stroke in the INSTRUCT (International stroke outcomes sTudy): a meta-analysis of individual participant data. Circ Cardiovasc Qual Outcomes 2017;10.

3. Gall SL, Donnan G, Dewey HM, et al. Sex differences in presentation, severity, and management of stroke in a population-based study. Neurology 2010;74:975-81.

4. Lisabeth LD, Brown DL, Morgenstern LB. Barriers to intravenous tissue plasminogen activator for acute stroke therapy in women. Gend Med 2006;3:270-8.

5. Reeves MJ, Bushnell CD, Howard G, et al. Sex differences in stroke: epidemiology, clinical presentation, medical care, and outcomes. Lancet Neurol 2008;7:915-26.

6. Etherton MR, Wu O, Cougo P, et al. Integrity of normal-appearing white matter and functional outcomes after acute ischemic stroke. Neurology 2017;88:1701-8.

7. Rost NS, Cougo P, Lorenzano S, et al. Diffuse microvascular dysfunction and loss of white matter integrity predict poor outcomes in patients with acute ischemic stroke. $J$ Cereb Blood Flow Metab 2018;38:75-86

8. Wardlaw JM, Doubal FN, Valdes-Hernandez M, et al. Blood-Brain barrier permeability and long-term clinical and imaging outcomes in cerebral small vessel disease. Stroke 2013:44:525-7.

9. Arsava EM, Rahman R, Rosand J, et al. Severity of leukoaraiosis correlates with clinical outcome after ischemic stroke. Neurology 2009;72:1403-10.

10. Wardlaw JM, Smith EE, Biessels GJ, et al. Neuroimaging standards for research into small vessel disease and its contribution to ageing and neurodegeneration. Lancet Neurol 2013;12:822-38.

11. Etherton MR, Wu O, Cougo P, et al. Structural integrity of normal appearing white matter and sex-specific outcomes after acute ischemic stroke. Stroke 2017:48:3387-9.

12. Rosenberg GA, Estrada EY, Dencoff JE. Matrix metalloproteinases and TIMPs are associated with blood-brain barrier opening after reperfusion in rat brain. Stroke 1998;29:2189-95.

13. Corbin ZA, Rost NS, Lorenzano S, et al. White matter hyperintensity volume correlates with matrix metalloproteinase-2 in acute ischemic stroke. J Stroke Cerebrovasc Dis 2014;23:1300-6.

14. Lorenzano S, Rost NS, Khan M, et al. Oxidative stress biomarkers of brain damage: hyperacute plasma F2-isoprostane predicts infarct growth in stroke. Stroke 2018;49:630-7.

15. Sorensen AG, Wu O, Copen WA, et al. Human acute cerebral ischemia: detection of changes in water diffusion anisotropy by using MR imaging. Radiology 1999;212:785-92.

16. Boxerman JL, Schmainda KM, Weisskoff RM. Relative cerebral blood volume maps corrected for contrast agent extravasation significantly correlate with glioma tumor grade, whereas uncorrected maps do not. AJNR Am J Neuroradiol 2006;27:859-67.

17. Wu O, Ostergaard L, Weisskoff RM, et al. Tracer arrival timinginsensitive technique for estimating flow in Mr perfusion-weighted imaging using singular value decomposition with a block-circulant deconvolution matrix. Magn Reson Med 2003;50:164-74.

18. Copen WA, Deipolyi AR, Schaefer PW, et al. Exposing hidden truncation-related errors in acute stroke perfusion imaging. AJNR Am J Neuroradiol 2015;36:638-45.
19. Rost NS, Sadaghiani S, Biffi A, et al. Setting a gold standard for quantification of leukoaraiosis burden in patients with ischemic stroke: the Atherosclerosis risk in Communities study. J Neurosci Methods 2014:221:196-201.

20. Ay H, Benner T, Arsava EM, et al. A computerized algorithm for etiologic classification of ischemic stroke: the causative classification of stroke system. Stroke 2007;38:2979-84.

21. Bruno A, Shah N, Lin C, et al. Improving modified Rankin scale assessment with a simplified questionnaire. Stroke 2010;41:1048-50.

22. Ferguson KJ, Wardlaw JM, Edmond CL, et al. Intracranial area: a validated method for estimating intracranial volume. $J$ Neuroimaging 2005;15:76-8.

23. Tong DC, Yenari MA, Albers GW, et al. Correlation of perfusion- and diffusion-weighted MRI with NIHSS score in acute (. Neurology 1998:50:864-70.

24. Lovblad KO, Baird AE, Schlaug G, et al. Ischemic lesion volumes in acute stroke by diffusion-weighted magnetic resonance imaging correlate with clinical outcome. Ann Neurol 1997;42:164-70.

25. Topakian R, Barrick TR, Howe FA, et al. Blood-Brain barrier permeability is increased in normal-appearing white matter in patients with lacunar stroke and leucoaraiosis. J Neurol Neurosurg Psychiatry 2010;81:192-7.

26. Arba F, Leigh $R$, Inzitari D, et al. Blood-Brain barrier leakage increases with small vessel disease in acute ischemic stroke. Neurology 2017;89:2143-50.

27. Wardlaw JM, Doubal F, Armitage $P$, et al. Lacunar stroke is associated with diffuse blood-brain barrier dysfunction. Ann Neurol 2009;65:194-202.

28. Bang OY, Saver JL, Alger JR, et al. Patterns and predictors of bloodbrain barrier permeability derangements in acute ischemic stroke. Stroke 2009;40:454-61.

29. Wilson AC, Clemente L, Liu T, et al. Reproductive hormones regulate the selective permeability of the blood-brain barrier. Biochim Biophys Acta 1782;2008:401-7.

30. Maggioli E, McArthur S, Mauro C, et al. Estrogen protects the blood-brain barrier from inflammation-induced disruption and increased lymphocyte trafficking. Brain Behav Immun 2016:51:212-22.

31. Munoz Maniega S, Chappell FM, Valdes Hernandez MC, et al. Integrity of normal-appearing white matter: influence of age, visible lesion burden and hypertension in patients with small-vessel disease. J Cereb Blood Flow Metab 2017;37:644-56.

32. Farrall AJ, Wardlaw JM. Blood-Brain barrier: ageing and microvascular disease-systematic review and meta-analysis. Neurobiol Aging 2009;30:337-52.

33. Simpkins AN, Dias C, Leigh R. National Institutes of health natural history of stroke I. Identification of reversible disruption of the human blood-brain barrier following acute ischemia. Stroke 2016;47:2405-8

34. Leigh R, Christensen S, Campbell BC, et al. Pretreatment blood-brain barrier disruption and post-endovascular intracranial hemorrhage. Neurology 2016;87:263-9.

35. Liu C, Yan S, Zhang R, et al. Increased blood-brain barrier permeability in contralateral hemisphere predicts worse outcome in acute ischemic stroke after reperfusion therapy. J Neurointerv Surg 2018.

36. Vos SB, Jones DK, Viergever MA, et al. Partial volume effect as a hidden covariate in DTI analyses. Neurolmage 2011;55:1566-76.

37. Liu HL, YY W, Yang WS, et al. Is Weisskoff model valid for the correction of contrast agent extravasation with combined T1 and T2* effects in dynamic susceptibility contrast MRI? Med Phys 2011;38:802-9

38. Skinner JT, Moots PL, Ayers GD, et al. On the use of DSC-MRI for measuring vascular permeability. AJNR Am J Neuroradiol 2016;37:80-7.

39. Villringer K, Sanz Cuesta BE, Ostwaldt AC, et al. Dce-Mri bloodbrain barrier assessment in acute ischemic stroke. Neurology 2017;88:433-40.

40. Bonekamp D, Deike K, Wiestler B, et al. Association of overall survival in patients with newly diagnosed glioblastoma with contrastenhanced perfusion MRI: Comparison of intraindividually matched T1 - and T2 (*) -based bolus techniques. J Magn Reson Imaging 2015;42:87-96. 